\title{
Lateral Deformation of a Silicon Crystal Surface Structure Induced by Low-Fluence Ion-Beam Irradiation
}

\author{
Xiaowei Guo \\ Department of Intelligent Mechanical Engineering, \\ Kochi University of Technology, Tosayamada, Kami-city, Kochi 782-8502, Japan \\ Sadao Momota* \\ School of Environmental Science and Engineering, \\ Kochi University of Technology, Tosayamada, Kami-city, Kochi 782-8502, Japan \\ Noriko Nitta \\ Center for Nanotechnology, Research Institute, Kochi University of Technology, \\ Tosayamada, Kami-city, Kochi 782-8502, Japan \\ Kazuki Maeda \\ Department of Intelligent Mechanical Engineering, \\ Kochi University of Technology, Tosayamada, Kami-city, Kochi 782-8502, Japan \\ (Received 20 December 2014; Accepted 26 January 2015; Published 14 February 2015)
}

\begin{abstract}
The increasing importance of the volume expansion effect on crystalline materials induced by ion-beam irradiation has drawn much attention because of its applications. For example, the expansion effect is used as a good probe to investigate any crystalline-amorphous (c-a) phase change and/or damage. Because the expansion rate and its depth profile can be controlled by means of the irradiation parameters, such as fluence and energy, the deformation of structures on the micro-/nano-meter scale is expected. The fluence needed to achieve deformation is relatively low, and it is expected that irradiation-induced damage is reduced compared with that induced by conventional ion-beam fabrication. Therefore, this expansion effect is a potential method to improve the ion-beam technology employed to fabricate complicated 3-dimensional structures requested in actively developing industrial fields, such as MEMS/NEMS. [DOI: 10.1380/ejssnt.2014.35]
\end{abstract}

Keywords: Ion implantation; Order-disorder phase transition; Radiation damage; Nano structure processing; Silicon; Lateral deformation

\section{INTRODUCTION}

In recent studies, the expansion effect has been observed in various crystals $[1,4-9]$, and the controllability along the vertical direction, a swelling height, was confirmed [10]. In contrast, no remarkable deformation of a crystalline silicon (c-Si) nano-structure along the lateral direction has been observed. Upon irradiating an ion beam on an amorphous silicon (a-Si) micro-structure, an irreversible deformation along the lateral direction has been observed $[11,12]$. However, a high fluence, which is required for an irreversible deformation, would lead to the prolongation of the processing time compared with a deformation arising from the expansion effect. Furthermore, more studies are necessary to understand the expansion effect.

The deformation of a micro/nano-meter scale structure of c-Si along the lateral direction, which is induced by Kr-beam irradiation, was investigated. As the first step of the investigations, the present study focuses on the contributions of the fluence and the structure size. And the lateral deformation effect is discussed based on the crystalline-amorphous (c-a) phase change obtained from Raman spectroscopy and TEM observations. Furthermore, the present results will lead to a further understanding of the ion-beam induced expansion effect in crystalline materials.

\footnotetext{
* Corresponding author: momota.sadao@kochi-tech.ac.jp
}

\section{EXPERIMENTAL}

\section{A. Preparation of initial nanostructures}

Czochralski-grown (Cz-Si) p-type $\mathrm{Si}\langle 100\rangle$ samples, whose size and thickness is $1.5 \times 1.5 \mathrm{~cm}^{2}$ and $0.5 \mathrm{~mm}$, were used in the present studies. In order to clean and remove any oxidized layer of the sample surface, all of the samples were sequentially washed in acetone, ultra-pure water, $18 \%$ buffered hydrofluoric acid (BHF) and ultrapure water. An initial pattern of stripe structure was fabricated on the cleaned c-Si surface by using a $30 \mathrm{keV}$ $\mathrm{Ga}^{+}$FIB (QUANTA 3D 200i dual-beam system, FEI). An SEM image and a schematic picture of the cross section of the stripe structure are shown in Fig. 1. The length, $\mathrm{L}$, and the height, $\mathrm{H}$, of the stripe, which are defined in Fig. 1, are $10 \mu \mathrm{m}$ and $\sim 500 \mathrm{~nm}$, respectively. Stripe structures, which consist a pile with four different widths of about 200, 300, $400 \mathrm{~nm}$ and $500 \mathrm{~nm}$, were prepared. The formation of a damaged layer on the surface of a nano-structure, introduced by the FIB process, was reported in Ref. [13]. In order to remove this layer, processed samples were etched with $18 \%$ BHF for 12 minutes under an ultrasonic condition at room temperature. In order to relax any possible residual damage, such as point defects, we annealed samples in an argon ambient environment at atmospheric pressure by desktop lamp heating (MILA-3000, ULAVC). The condition of the annealing process was the same as that described in Ref. [14]. 


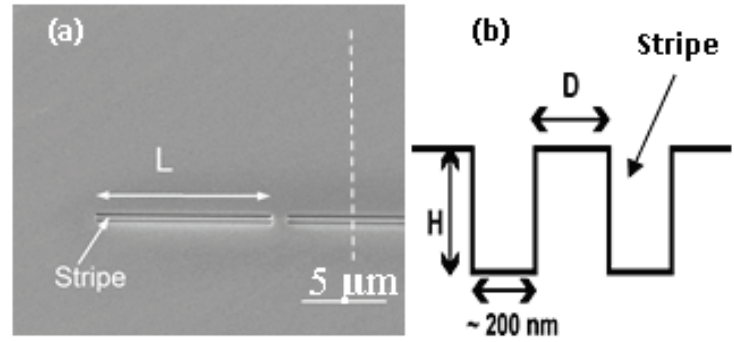

FIG. 1. (a) SEM image of the stripe structure and (b) schematic diagram of its cross section.

\section{B. Modification of initial nanostructures by Kr-beam irradiation}

In order to generate the lateral deformation in initial structures, $\mathrm{Kr}^{+2}$ beams with an energy of $240 \mathrm{keV}$ were implanted at room temperature with an incident angle of $5^{\circ}$ so as to avoid any channeling effect. Kr beams were prepared by a $10-\mathrm{GHz}$ NANOGAN, which is an ECR ion source installed at Kochi University of Technology [15]. The fluence of the $\mathrm{Kr}$ beam was $6,8,10,30$, and $50 \times$ $10^{13}$ ions $/ \mathrm{cm}^{2}$.

\section{Evaluation of the defect distribution by SRIM}

According to previous studies[10], the expansion effect shows a remarkable relation to damages induced by ionbeam implantation. In order to evaluate the spacial distribution of damages, SRIM-2008 [16] was used in a "detailed calculation with full damage cascades" mode for 10000 projectiles of the Kr-ion with kinetic energies of 240 $\mathrm{keV}$. The calculated distributions of vacancies and ions are shown in Fig. 2 and Fig. 3, respectively. This calculation does not consider the self-annealing effect and the nonlinear effect, such as the interactions between defects. Figure 2 shows that the distribution of the vacancy is close to a Gaussian-like distribution. The distribution of vacancies covers the depth range from $0 \mathrm{~nm}$ to $300 \mathrm{~nm}$, and shows its maximum at a depth of about $100 \mathrm{~nm}$. As shown in Fig. 3, the range distribution of implanted Kr-ion also shows a Gaussian-like distribution, which is characterized by the projection range, $142.6 \mathrm{~nm}$, and struggling, 44.9 nm. The calculated distribution shows that the major part of Kr-ions, $\sim 75 \%$, stops at a depth range between $100 \mathrm{~nm}$ and $190 \mathrm{~nm}$. According to the above consideration, the region of interests, which would contribute to the expansion effect, is the range of depth between $0 \mathrm{~nm}$ and $200 \mathrm{~nm}$.

\section{Evaluation of the lateral deformation and c-a phase change of an irradiated Si sample}

In order to obtain the lateral deformation of a stripe structure, FE-SEM (JSM-7401F, JEOL) was utilized to measure the geometrical size of the structures both before and after Kr-beam irradiation. The cross-section, which

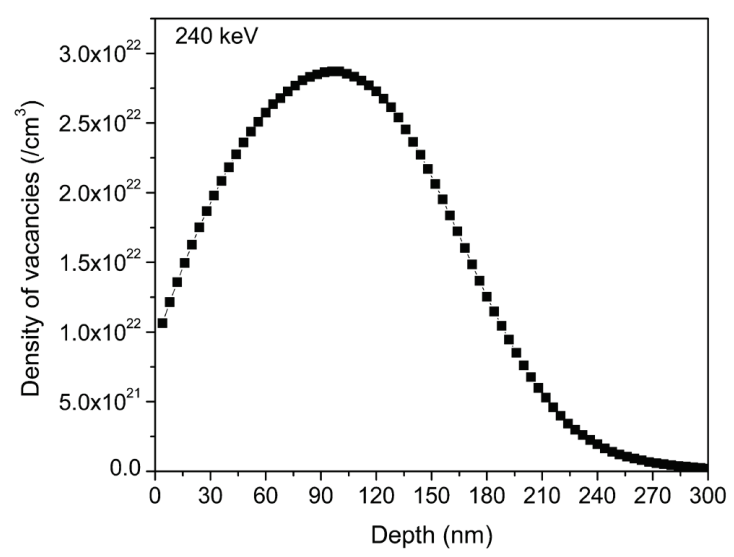

FIG. 2. Distribution of the vacancies in c-Si induced by a 240 keV Kr-beam calculated by SRIM.

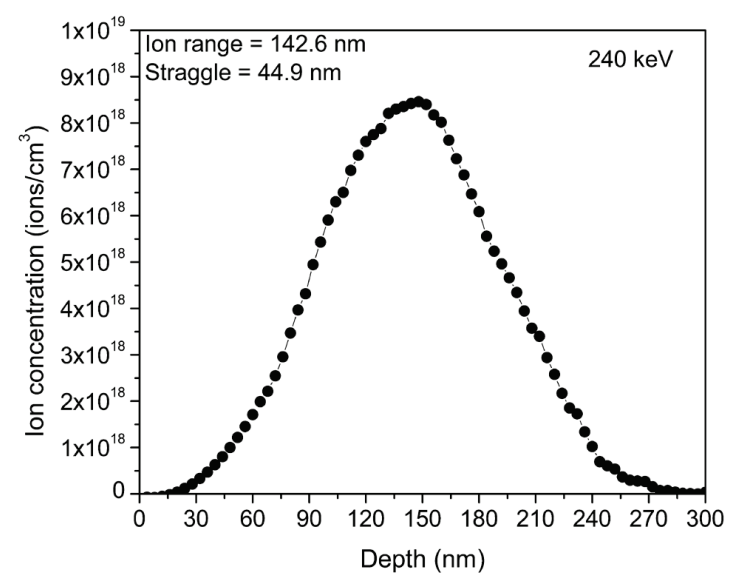

FIG. 3. Range distribution of a Kr-beam with $240 \mathrm{keV}$ in c-Si calculated by SRIM.

was formed by a cutting to cross the stripe pattern vertically, was observed. In the case of the deformation of crystalline materials induced by low-fluence ion-beam irradiation, it is expected that the evolutional transition of matrix structure, such as the growing features of the amorphous phase, plays an important role. In order to obtain information on the amorphization effect, a Raman spectrometer (HR 800 Horiba, $532 \mathrm{~nm}$ of laser) and a TEM (2100F, JEOL) were applied.

\section{RESULTS AND DISCUSSIONS}

\section{A. Observation of stripe structures by SEM}

Typical SEM images of stripe structures before and after Kr-beam irradiation are shown in Fig. 4. Figure 4(a) shows the cross section of a center pile in stripe structure at a given cutting plane before Kr-beam irradiation. The slope of sidewalls and curvature at the top edges is found in the observed cross sections. It is considered that those features arise from preparation processes of the initial structure. The cross section of the stripe structure 

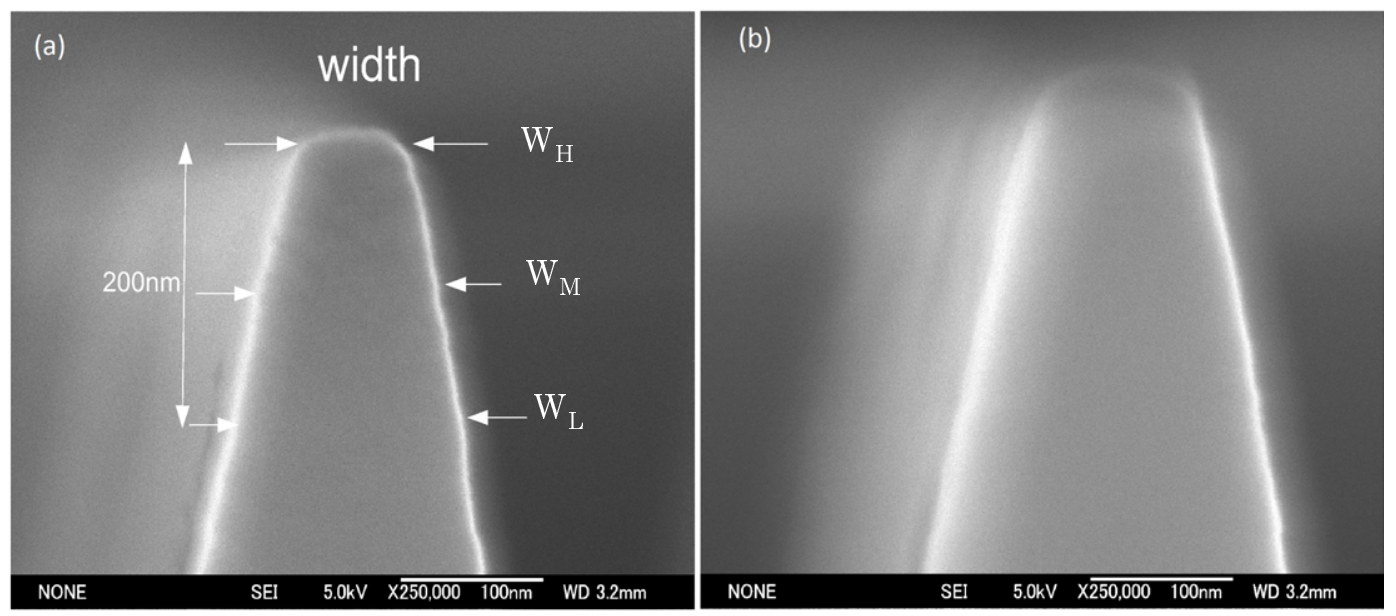

FIG. 4. SEM images of the stripe structures. (a) and (b) show cross sections of the center piles in stripe structures before and after the irradiation of $\mathrm{Kr}$ beams with $240 \mathrm{keV}, 8 \times 10^{13}$ ions $/ \mathrm{cm}^{2}$.

after Kr-beam irradiation is shown in Fig. 4(b) and the remarkable lateral deformation is identified as an increase in the width of a center pile, compared with Fig. 4(a). Therefore, the present study is on the deformation of stripe structure with a taper-shaped cross section.

\section{B. Lateral deformation of the irradiated stripe structures}

The widths of the center piles in stripe structures were measured at three different levels: higher $\left(\mathrm{W}_{\mathrm{H}}\right)$, middle $\left(\mathrm{W}_{\mathrm{M}}\right)$ and lower $\left(\mathrm{W}_{\mathrm{L}}\right)$ positions, which are defined in Fig. 4(a) according to consideration of the SRIM calculation in section IID. Because of a curvature at the top edge, relatively large ambiguities were observed in $\mathrm{W}_{\mathrm{H}}$. Therefore, the widths $W_{M}$ and $W_{L}$ are used in the following discussion. As the central value, the average of $\mathrm{W}_{\mathrm{M}}$ and $W_{L}$ is employed. The relation between the change in the widths, measured before and after Kr-beam irradiation, and the fluence of Kr-beam for structures with the width of $100 \mathrm{~nm} \sim 200 \mathrm{~nm}$ is shown in Fig. 5. The width change, which is provided from one sample, is shown with no error bars. As shown in Fig. 5, the remarkable fluence dependence of the lateral deformation was observed for stripe structures. The width change increases up to a fluence of $8 \times 10^{13}$ ions $/ \mathrm{cm}^{2}$, and decreases to almost zero at a fluence of $5 \times 10^{14}$ ions $/ \mathrm{cm}^{2}$. To avoid any contribution of ambiguity in the initial structure size induced by the etching process, the lateral expansion rate was calculated, as shown in Fig. 6. Since sidewalls of a center pile have a slope with a tilt angle of $70 \sim 80$ degree, densities of implanted ions and defects changes along a lateral direction even at the same level. This effect would relax the intrinsic level dependence and gives obvious contribution for structures with small width. Therefore, the expansion rates obtained for $\mathrm{W}_{\mathrm{M}}$ and $\mathrm{W}_{\mathrm{L}}$ are averaged and the error bars are defined by those differences. Calculated lateral expansion rate shows similar behavior with the width change, and becomes maximum value of $30 \%$ at a fluence of $8 \sim 10 \times 10^{13}$ ions $/ \mathrm{cm}^{2}$.

In order to investigate the contribution of the structure

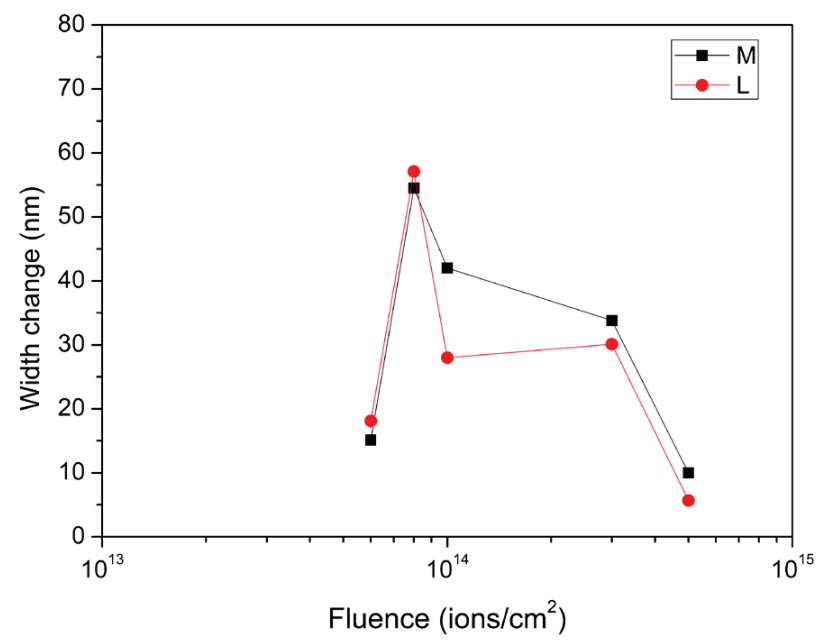

FIG. 5. Fluence dependence of observed lateral deformation. The width change of the center pile of stripe structures with an initial width of $100 \mathrm{~nm} \sim 200 \mathrm{~nm}$ is shown in this figure.

size, the width change of the center pile in stripe structures was observed as a function of the width of the center pile at $8 \times 10^{13}$ ions $/ \mathrm{cm}^{2}$ and $1 \times 10^{14}$ ions $/ \mathrm{cm}^{2}$, at which a large lateral deformation effect was observed, as shown in Fig. 7. Figure 7(a) indicates that the width change becomes its maximum value, $55 \mathrm{~nm}$, and decreases with the lateral size. A similar behavior is also found in Fig. 7(b).

\section{Observation of Raman spectrum}

To investigate the inner change of c-Si induced by the Kr-beam irradiation, a Raman spectrum was observed at the flat region near the patterned region. Observed evolutional behavior of the Raman spectrum with the fluence of the Kr-beam is shown in Fig. 8. A sharp peak at $520 \mathrm{~cm}^{-1}$, which corresponds to c-Si, shrinks with the fluence. On the other hand, a peak at $510 \mathrm{~cm}^{-1}$, which corresponds to a defective crystalline phase, appears in 


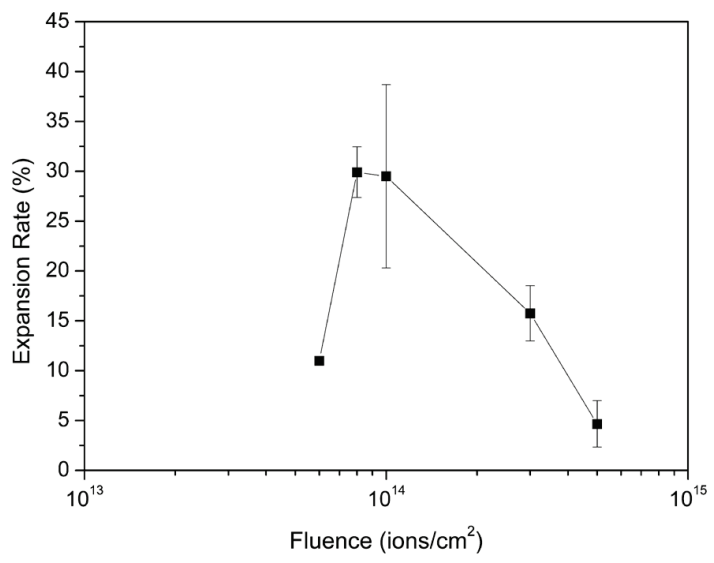

FIG. 6. Fluence dependence of the lateral expansion rate of stripe structures with a width of a central pile of $100 \mathrm{~nm} \sim$ $200 \mathrm{~nm}$.

the spectrum for higher fluence. Also the broad peak placed at $480 \mathrm{~cm}^{-1}$, which corresponds to a-Si, grows with the fluence, and becomes the dominant component in the spectrum for a fluence larger than $3 \times 10^{14}$ ions $/ \mathrm{cm}^{2}$.

The crystalline volume fraction of a-Si sample deduced from the Raman spectrum, $\phi_{c}$, was introduced in [17] as

$$
\phi_{c}=\left(I_{520}+I_{510}\right) /\left(I_{520}+I_{510}+I_{480}\right),
$$

where $I_{i}$ is the area under the Gaussian distribution, whose center is located at $\mathrm{i}$. As a semi-quantitative index, the amorphization faction, $\phi_{a}$, is defined using $\phi_{c}$ as

$$
\phi_{a}=I_{480} /\left(I_{520}+I_{510}+I_{480}\right) .
$$

In Fig. 9, the amorphization fraction, calculated from the deconvoluted spectra obtained from three-peak fitting of the observed Raman spectrum, is shown as a function of fluence of the Kr-beam. The figure indicates that the ca phase change proceeds continuously in the fluence range between $6 \times 10^{13}$ ions $/ \mathrm{cm}^{2}$ and $5 \times 10^{14}$ ions $/ \mathrm{cm}^{2}$. Considering Fig. 5, the range of the Kr-beam fluence, at which the lateral deformation effect shows remarkable fluence dependence, corresponds to the transitional range from the crystal phase to amorphous phase. The lateral deformation achieves the maximum at an amorphous fraction of about $85 \%$. Also at higher fluence, the lateral deformation relaxes and returns to zero at an amorphous fraction of about $100 \%$. As a result, Raman spectroscopy indicates that the amorphization process of $\mathrm{c}-\mathrm{Si}$ has an obvious relation with the lateral deformation of the c-Si nano-structure.

\section{Observation by TEM}

Figure 10 shows cross-sectional TEM images of the stripe structure irradiated by a Kr beam with three different fluences. In this figure, an amorphous layer is clearly recognized in the top and sidewalls of the stripe structure, and is separated from the crystalline region by an interface. The thickness of the amorphous layer grows with the Kr-beam fluence and reaches to $270 \mathrm{~nm}$ at the top
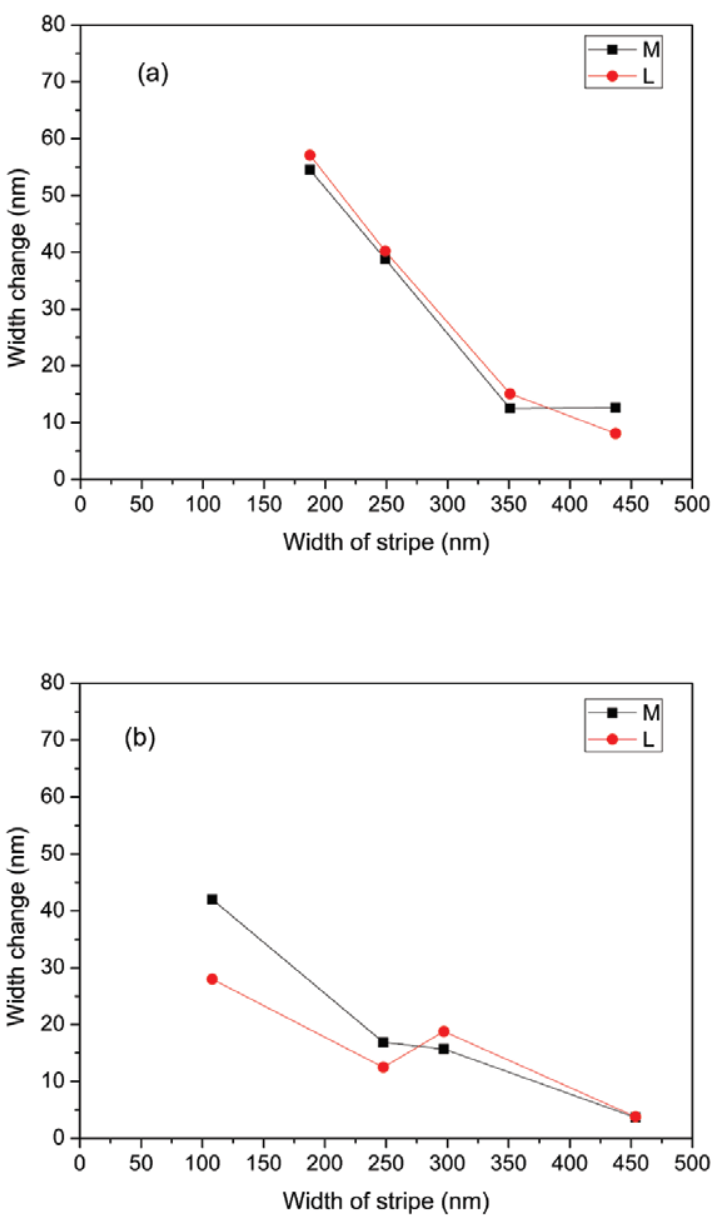

FIG. 7. Structure size dependence of the lateral deformation. (a) and (b) show the observed results with a fluence of $8 \times 10^{13}$ and $1 \times 10^{14}$ ions $/ \mathrm{cm}^{2}$, respectively.

of the center pile and $249 \mathrm{~nm}$ at the flat region for a fluence of $5 \times 10^{14}$ ions $/ \mathrm{cm}^{2}$. This result indicates that the depth region of interest, $0-200 \mathrm{~nm}$, mentioned in II D, is completely amorphized for higher fluence. The growing nature of the amorphous layer is consistent with the increase in the amorphous fraction, which was obtained from the Raman spectra. The figure also shows the differences in the thickness of the amorphous layer at the right and left sidewalls of the stripe structure. The tilt angle of Kr-beam irradiation from the normal of Si-surface would cause the asymmetric feature. In section III C, observed Raman spectra indicated the importance of the c-a phase change in order to understand the lateral deformation effect on nano-structure. In the case of heavy-ion irradiation at low temperature, the c-a phase change proceeds through the overlap of the isolated damaged region induced by ion-beam irradiation, and shows a heterogeneous nature. Therefore, the amorphization induced by Kr-ion irradiation at room temperature is expected to be heterogeneous. Also, observed lateral deformation indicates structure size dependence, as described in section IIIB. To investigate the heterogeneous features in and around the amorphous region and contributions of the lateral size, high-resolution TEM images of center piles were observed for two structures with different lateral sizes, and shown 


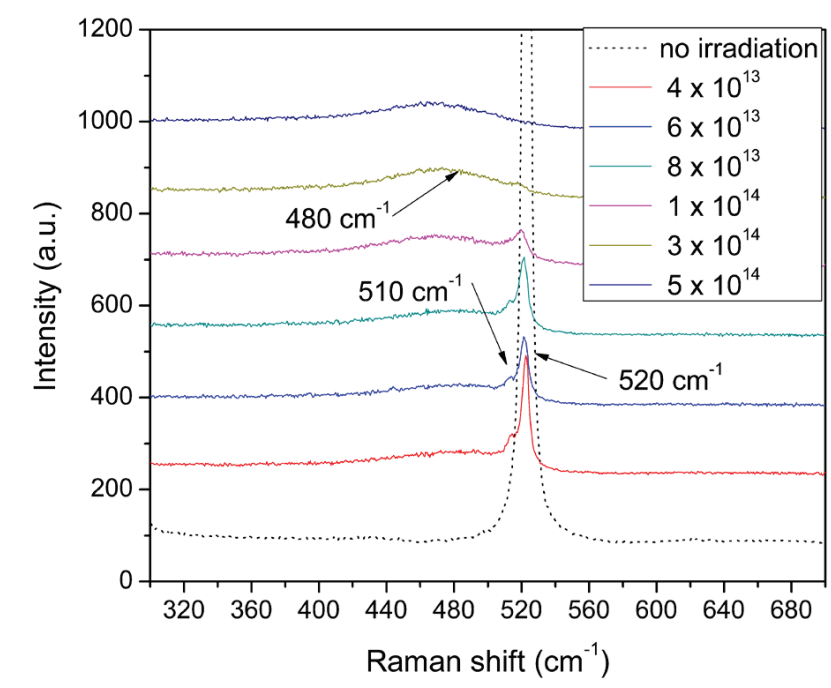

FIG. 8. Raman spectra of Si irradiated by Kr beam. The spectra for a virgin crystal $\mathrm{Si}$ is also shown for reference. The spectrum for each fluence is vertically displaced for clarity.

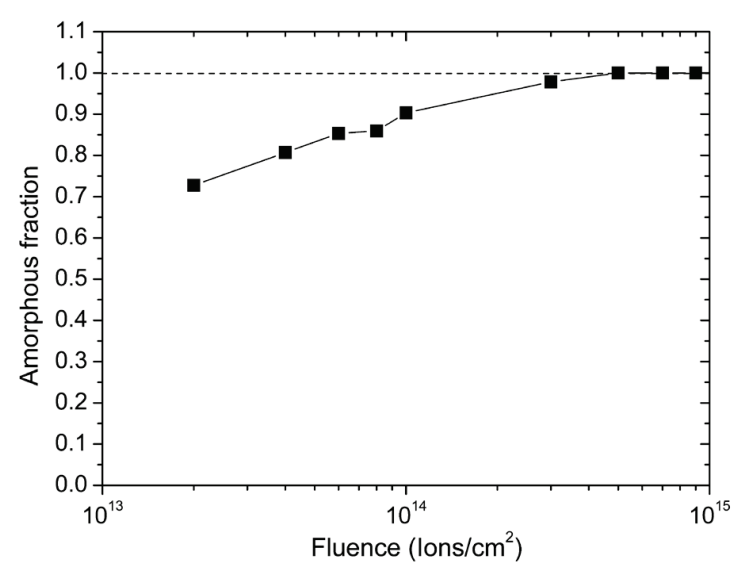

FIG. 9. Growth of amorphous fraction with the fluence of the Kr beam.

in Figs. 11 and 12.

Figure 11(b), which is high-resolution image indicated in Fig. 11(a), shows an interface in the stripe structure with the width of the center pile of $\sim 100 \mathrm{~nm}$ irradiated with a fluence of $1 \times 10^{14}$ ions $/ \mathrm{cm}^{2}$. The width of the crystalline region, which is located between two interfaces in the center pile, is about $40 \mathrm{~nm}$. In Fig. 11(c), which is a higher resolution image around the interface region, crystalline (amorphous) pockets, highlighted by black (white) circles in Fig. 11(c), are found in the amorphous (damaged crystalline) region as in Ref. [18]. According to Ref. [18], c-a phase change induced by ion beam irradiation was discussed by means of amorphous pockets and crystalline pockets, which were formed in semi-continuous amorphous layer due to solidification. A damaged crystalline region is confirmed to be the coexistence of the diffraction pattern and the continuous circle in Fig. 11(d).

Fig. 12(a) shows the cross section of the stripe structure with the width of the center pile of $\sim 450 \mathrm{~nm}$ irradiated with a fluence of $1 \times 10^{14}$ ions $/ \mathrm{cm}^{2}$. The width of the crystalline region between the two interfaces is $\sim 300 \mathrm{~nm}$, which is much larger than that of the width of $\sim 100 \mathrm{~nm}$. Fig. 12(b) is a high-resolution image of the interface region indicated in Fig. 12(a). Fig. 12(c), observed around the interface region, shows an existence of crystalline (amorphous) pockets, highlighted by black (white) circle, as in Fig. 11(c). A continuous circle found in Fig. 12(e), which is a diffraction pattern obtained in the region defined in Fig. 12(b), indicates incomplete amorphization and the existence of a strain in the system. In contrast, the diffraction pattern, which is obtained from Fig. 12(d), in Fig. 12(f) shows that the region under the interface keeps the crystal nature.

In both TEM images of Fig. 11 and Fig. 12, crystalline/amorphous pockets, which indicated heterogeneous amorphization, are found in the vicinity of the interface. And the width of the crystalline region, which is placed between two interfaces in the center pile, changes according to the lateral size of the stripe structure. Decreasing the lateral size, the two interfaces approach to remove the crystal region in the center pile.

\section{E. Mechanism of lateral deformation induced by the low-fluence $\mathrm{Kr}$-beam irradiation}

The sputtering effect is another important contribution to cause the lateral deformation. Considering the sputtering rate and its incident angle dependence, which are evaluated by SRIM, the contribution of sputtering effect to the lateral deformation under the present irradiation condition is calculated to be less than $0.64 \mathrm{~nm}$ even at the maximum fluence of $5 \times 10^{14}$ ions $/ \mathrm{cm}^{2}$. Therefore, the sputtering effect is not considered in the following argument.

The presently observed deformation, which was induced by Kr-beam irradiation with low fluence, is compared with the previously observed deformation. The growing and shrinking of the lateral deformation was observed in the fluence range, within which the crystalline phase continuously changes to the amorphous phase. The effective fluence range for the lateral deformation, is significantly lower than that for irreversible deformation, which was observed in a-Si micro/nano-structure[11, 12]. The irreversible deformation for amorphous material is called plastic deformation, and is successfully explained by a viscoelastic model proposed by Trinkaus and Ryaznov [19]. And, the presently observed deformation is promoted for structures with small lateral size, and suppressed for those with large lateral size. The size effect on plastic deformation was observed for spherical $\mathrm{SiO}_{2}$ colloid [20] and ascribed to a nonhydrostatic stress distribution by surface curvature. Because initial phases and/or morphological shapes as well as irradiation parameters are different, it is difficult to apply those previous models to explain the presently observed lateral deformation. In the present studies, initial structures are in the crystalline phase as described in section IIID. The amorphous/crystalline pockets and internal stress were observed in TEM images for stripe structures, in which a remarkable lateral deformation was observed. Therefore, the fluence dependence of the lateral deformation is considered by means of the amorphous/crystalline pockets. 


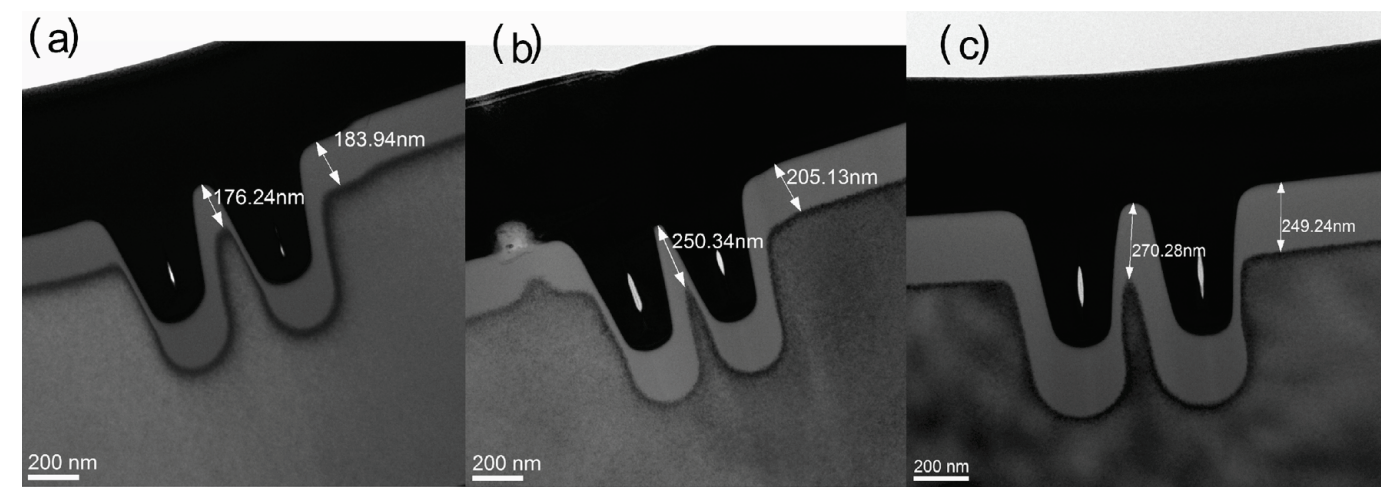

FIG. 10. Cross-sectional TEM images of Stripe structures irradiated by a Kr beam with fluences of (a) $8 \times 10^{13}$, (b) $1 \times 10^{14}$ and (c) $5 \times 10^{14}$ ions $/ \mathrm{cm}^{2}$.

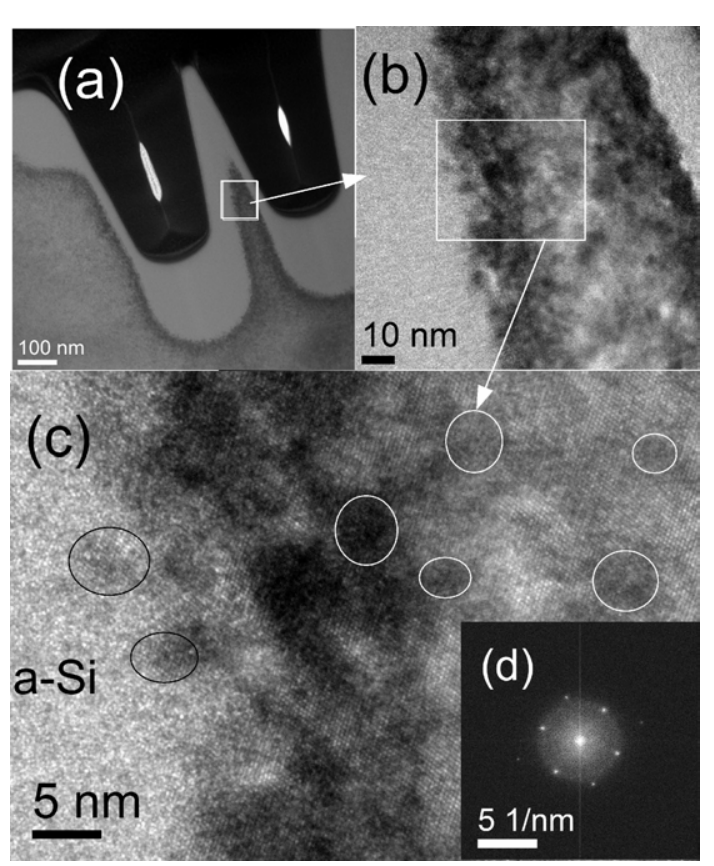

FIG. 11. TEM images of stripe structures with the width of the center pile of $\sim 100 \mathrm{~nm}$, irradiated with a fluence of $1 \times 10^{14}$ ions $/ \mathrm{cm}^{2} ;$ (a) cross-sectional image; (b), (c) are images of higher resolution. The rectangles in (a) and (b) indicate the observed regions for higher resolution. Amorphous pockets and crystalline pockets are highlighted by white circles and black circles, respectively. (d) Fourier transform of (c).

The cascade localization induced bias (CLIB) model, proposed by Yoshiie et al. [21] to explain the behaviors of defects in the lattice system, indicates that defects induced in the Si lattice by Kr-beam irradiation would be absorbed by boundaries between amorphous/crystalline pockets and their surroundings. This absorption stimulates the growth of amorphous pockets and shrinkage of the crystalline pockets. Also, when amorphous pockets grow to overlap with others, amorphous pockets combine and contributions of boundaries to absorb defects are suppresses. Based on this idea, the fluence dependence can be qualitatively understood as follows. In the case of lower fluence, amorphous pockets are produced and grow to promote a heterogeneous nature with the fluence of $\mathrm{Kr}$ beam.
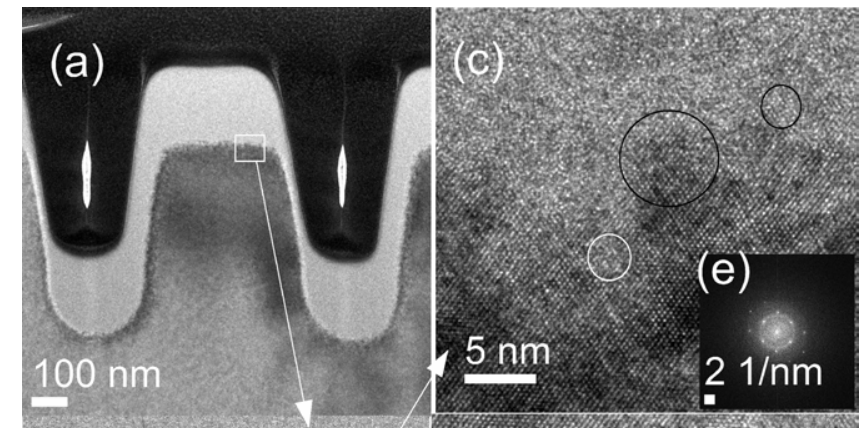

(b)

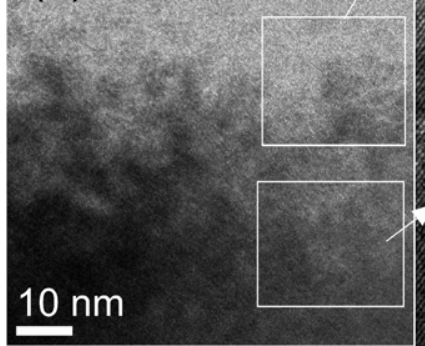

(d)

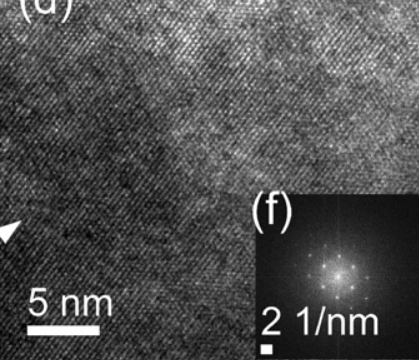

FIG. 12. TEM images of stripe structure with the width of the center pile of $\sim 450 \mathrm{~nm}$, irradiated with a fluence of $1 \times$ $10^{14}$ ions $/ \mathrm{cm}^{2}$; (a) cross sectional image of the center pile; (b), (c), (d) are images in higher resolution; (e) and (f) are Fourier transform of (c) and (d), respectively.

Then the internal stress, which promotes the expansion effect, increases. On the other hand, in the case of higher fluence, amorphous pockets start combining with others with increasing the fluence of $\mathrm{Kr}$ beam. Then the internal stress decreases.

Considering observed TEM images, the following naive model is a possible candidate to explain the contribution of lateral size to the lateral deformation. In the case of a smaller lateral size, the crystalline region between two interfaces in the center pile reduces, and the major part of the center pile is occupied by the amorphous region. Therefore, contributions of stress induced by the heterogeneous nature would become obvious in the whole region between the interfaces in the left and right sidewall, and promote an expansion along the lateral direction. In contrast, in the case of the larger lateral size, the contribu- 
tion of the crystalline region, which tends to keep its form, becomes prior. Therefore, the lateral deformation effect would be suppressed by the crystalline region compared with structures of smaller lateral size.

\section{CONCLUSIONS}

In conclusions, a deformation of taper-shaped Si structures along the lateral direction has been successfully achieved firstly by irradiating $\mathrm{Kr}$ beam of $240 \mathrm{keV}$. The observed results have shown that the lateral deformation depends on the lateral size of stripe structure as well as the fluence of $\mathrm{Kr}$ beam. The typical fluence of a 240 $\mathrm{keV} \mathrm{Kr-beam} \mathrm{employed} \mathrm{to} \mathrm{provide} \mathrm{lateral} \mathrm{deformation} \mathrm{is}$ about $1 \times 10^{14}$ ions $/ \mathrm{cm}^{2}$. This fluence is much lower than the typical fluence of ion beams, which is needed to provide plastic deformation in amorphous materials. The amorphous/crystalline pockets, which were observed with stress in the lattice structure, can provide a consistent qualitative explanation for observed lateral deformation and its fluence dependence. The suppression effect on the lateral deformation, which would be originated from crystalline region between two interfaces in the center pile, provides a possible explanation for the structure size dependence. However, further investigations are needed to confirm the reliability of this idea. In addition, the contributions of irradiation energy and ion species will be performed in next studies.

\section{ACKNOWLEDGMENTS}

We thank Professor Taniwaki for providing valuable suggestions. We are indebted to all colleagues of MOMOTA-lab for helping to perform experiments involving ion beam irradiation.
[1] Y. H. Li, Y. Q. Wang, J. A. Valdez, M. Tang, and K. E. Sickafus, Nucl. Instrum. Methods Phys. Res. B 274, 182 (2012).

[2] C. Trautmann, M. Boccanfuso, A. Benyagoub, S. Klaumünzer, K. Schwartz, and M. Toulemonde, Nucl. Instrum. Methods Phys. Res. B 191, 144 (2002).

[3] C. Ascheron, A. Schindler, R. Flagmeyer, and G. Otto, Nucl. Instrum. Methods Phys. Res. B 36, 163 (1989).

[4] R. Nipoti, E. Albertazzi, M. Bianconi, R. Lotti, G. Lulli, M. Cervera, and A. Carnera, Appl. Phys. Lett. 70, 3425 (1997).

[5] J. S. Custer, M. O. Thompson, D. C. Jacobson, J. M. Poate, S. Roorda, W. C. Sinke, and F. Spaepen, Appl. Phys. Lett. 64, 437 (1994).

[6] J. Costantini, C. Trautmann, L. Thomé, J. Jagielski, and F. Beuneu, J. Appl. Phys. 101, 073501 (2007).

[7] M. Gauneau, R. Chaplain, A. Rupert, Y. Toudic, R. Callec, and E. Andre, J. Appl. Phys. 73, 2501 (1993).

[8] T. Fa, L. Li, S. Yao, M. Wu, and S. Zhou, Chin. Phys. B 20, 056101 (2011).

[9] P. Giri, V. Raineri, G. Franzo, and E. Rimini, Phys. Rev. B 65, 012110 (2001).

[10] S. Momota, J. Zhang, T. Toyonaga, H, Terauchi, K. Maeda, J. Taniguchi, H. Takashi, M. Furuta, and T. Kawaharamura, J. Nanosci. Nanotechnol. 12, 552 (2012).
[11] T. van Dillen, M. J. A. de Dood, J. J. Penninkhof, A. Polman, S. Roorda, and A. M. Vredenberg, Appl. Phys. Lett. 84, 3591 (2004).

[12] M.-O. Ruault, F. Fortuna, H. Bernas, M. C. Ridgway, and J. S. Williams, Appl. Phys. Lett. 81, 2617 (2002).

[13] J. Yu, J. Liu, J. Zhang, and J. Wu, Mater. Lett. 60, 206 (2006).

[14] J. Zhang, Mechanism and application of morphological changes of Si crystal material induced by ion-beam (Kochi University of Technology, Kami-city, 2012).

[15] S. Momota, Y. Nojiri, M. Saihara, A. Sakamoto, H. Hamagawa, and K. Hamaguchi, Rev. Sci. Instrum. 75, 1497 (2004).

[16] James F. Ziegler, and J. P. Biersack, The Stopping and Range of Ions in Matter (Springer US, New York, 1985).

[17] C. Droz, E. Vallat-Sauvain, J. Bailat, L. Feitknecht, J. Meier, and A. Shah, Sol. Energ. Mat. Sol. Cells 81, 61 (2004).

[18] L. Romano, K. S. Jones, K. Sekar, and W. A. Krull, J. Vac. Sci. Technol. B 27, 597 (2009).

[19] H. Trinkaus and A. Ryazanov, Phys. Rev. Lett. 74, 5072 (1995).

[20] T. van Dillen, E. van der Giessen, P. Onck, and A. Polman, Phys. Rev. B 74, 132103 (2006).

[21] T. Yoshiie, Y. Satoh, S. Kojima, and M. Kiritani, J. Nucl. Mater. 179-181, Part 2, 954 (1991). 